\title{
An Evaluation of Beam-Damage Zone in Si Wafer Machined by Gatan MicroPREP ${ }^{\mathrm{TM}}$ Laser-Ablation
}

\author{
Wayne Zhao ${ }^{1}$, Corbin Bennett ${ }^{1}$, Pradip Sairam Pichumani ${ }^{1}$, Gerald Walker ${ }^{1}$, Kevin Eaton ${ }^{1}$, Michael \\ Hassel Shearer ${ }^{2}$, Laurent Dumas ${ }^{1}$, Irene Brooks ${ }^{1}$ and Ying Wang ${ }^{2}$ \\ 1. Physical Failure Analysis, Center for Complex Analysis, Characterization Group, Fab8, \\ GLOBALFOUNDRIES, Malta, NY. \\ 2. Gatan, Pleasanton, CA.
}

In synergy with the continuous miniaturization of semiconductor devices with nanometer transistors as the basic building blocks, teams of physical failure analysis (PFA) and transmission electron microscopy (TEM) in wafer-foundries have to constantly explore novel and more efficient approaches to effectively reduce turnaround and improve quality in TEM sample-preparation and Advanced TEM analysis. Benefits of these efforts are evident in the facilitation of various generations of technology node development, device characterizations, process development, product engineering, and integration for yield learning and yield enhancement[1 2].

Semiconductor devices built on Integrated Chips (IC) are basically a structure of complex composites, constructed by components with various material properties and different material removal rates, such as Si-based semiconductor, conductive interconnects, and various dielectric and insulators. Currently, several techniques for TEM-preparation are available and widely used for PFA purpose in waferfoundries for IC devices. Each has its unique pros and cons. When and where to apply them, depends on sophistication of each PFA task pertinent to specific area of interest (AOI). For examples, focus ion beam (FIB) with Gallium source are quick and effective to slice device with scales of few micron to nanometers, suitable for applications from front-end-of-line (FEOL) and back-end-of-line (BEOL). For further large microstructures such as Through-Silicon Vias (TSV) and Chip Package, newly commercialized Plasma FIBs have been introduced. Improved milling rates can be seen, tools with even faster removal rates are still required, e.g., to timely expose super-large cross-sections.

Gatan microPREPTM with picosecond Laser Ablation is the fastest milling tool, ideal for millimeter to micron-level applications [2 3]. To reliably extend its application into TEM-preparation for nanometer scale IC device, the battle is how to employ the two-edged sword, i.e., balance the fastest removal rate with the least beam damage to the feature desired. Therefore, in-depth understandings of the microPREP application window and its associated marginality are essential. Summarized here are our first evaluation of the Laser damage zone in Si-wafer, machined by microPREP. Figures-1a 1e are SEM and X-ray tomography characterizations for a box-cut on top of a Si-wafer. The squared shaped cave was machined by two steps, a primary box (rough cut) followed by a secondary box cut using slice by slice polishing, similar to the cleaning / polishing pattern in FIB. X-ray tomography (Figs-1d 1e) indicated that there is no hidden damage invisible by the SEM examination. The seemingly wide damage boundary from top-down SEM, was actually edge effect from the sidewall slope of the box-cut. Figures-2a 2f is a TEM-foil as-machined by the microPREP from a Si-wafer (Fig-1a) and one additional TEM-lamellar by FIB (Fig-2f), extracted perpendicular to Fig-1a. Figures $3 \mathrm{a} \sim 3 \mathrm{~h}$ are TEM/HRTEM along the boundary of the FIB-cut (site\#1 5) and the Laser-cut (site\#6 21), and corresponding diffraction, XEDS Qmaps and extracted profiles. Surface damage by the Laser-cut was $\sim 200 \mathrm{~nm}$ Si damage / amorphous layer, plus $\sim 500 \mathrm{~nm}$ oxide on top, vs. 30 50nm amorphous surface 
layer by FIB. The Laser damaged zone in pure Si-wafer appeared relatively localized, which is encouraging for random site TEM-prep (rough-cut by microPREP and final-mill by FIB). For sitespecific TEM-prep on patterned wafers, suggestions for future evaluation are to monitor the accuracy / repeatability and reliability of the Laser beam positioning against AOI, since no motorized stage available. After Laser-Ablation, the AOI was covered by a layer of surface oxide (up to half micron thick), how to effectively "see through" the oxide to pin-point the original AOI mark, is a challenge pending breakthrough, (e.g., maybe, to replace high pressure air with Argon to blow off the debris) [4].

\section{References:}

[1] W. Zhao et al, Microscopy \& Microanalysis 20 (S3) (2014), p.362.

[2] Y. Wang et al, IC Packaging, FCMN, (2017), in print.

[3] D. Elswick et al, FIB Workshop, Washington DC (2017).

[4] Thanks go to Andreas Meyer of GLOBALFOUNDRIES Fab1 for his insights and inspiring discussions, and Fab8 Management and Legal teams for supporting the publication clearance.
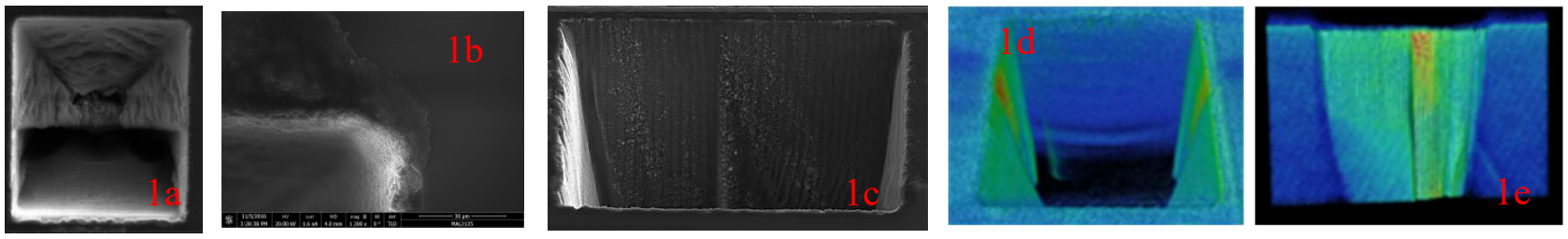

Figure 1. (a c) top-down SEM on the cut in Si wafer by microPREP; and X-ray Tomography (d e).
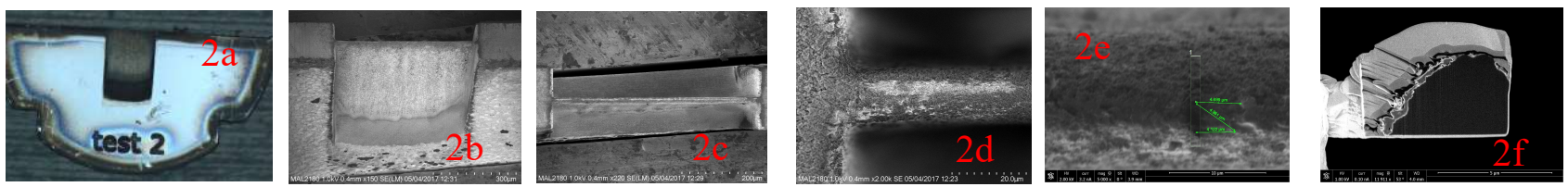

Figure 2. (a) Semi-TEM foil by microPREP; (b e) SEM for (a); and (f) $2^{\text {nd }}-$ TEM foil by FIB from (a).
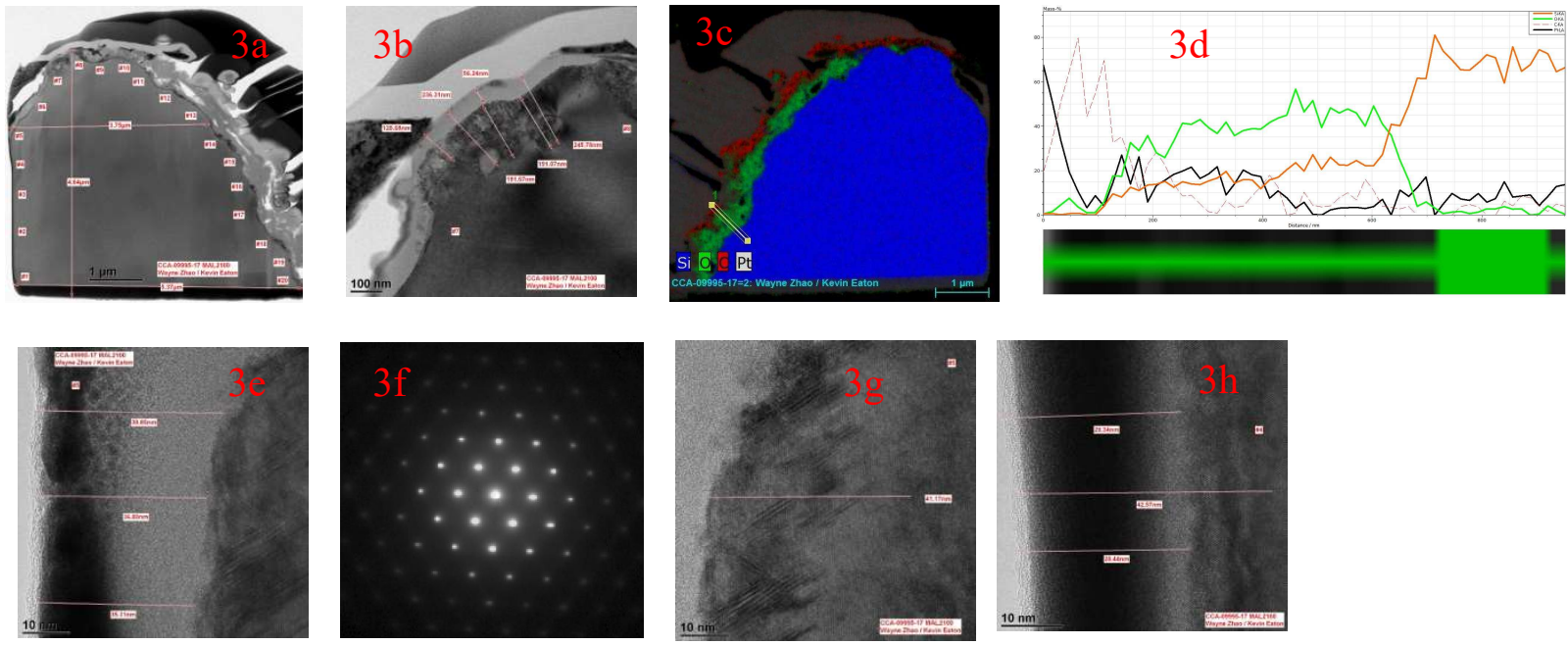

Figure 3. $(a \sim b)$ TEM for the $2^{\text {nd }}$ TEM foil and (c $\left.\sim d\right)$ XEDS mapping and extracted line profiles; and $(\mathrm{e} \sim \mathrm{h})$ HRTEM/Electron Diffraction for estimation of FIB-damage (e) vs. Laser-damage (g h) zone. 